\title{
Nonlinearities and effects of transverse beam size in beam position monitors
}

\author{
Sergey S. Kurennoy \\ Los Alamos National Laboratory, Los Alamos, New Mexico 87545
}

(Received 26 June 2001; published 13 September 2001)

\begin{abstract}
The fields produced by a long beam with a given transverse charge distribution in a homogeneous vacuum chamber are studied. Signals induced by a displaced finite-size beam on electrodes of a beam position monitor (BPM) are calculated and compared to those produced by a pencil beam. The nonlinearities and corrections to BPM signals due to a finite transverse beam size are calculated for an arbitrary chamber cross section. Simple analytical expressions are given for a few particular transverse distributions of the beam current in a circular or rectangular chamber. Of particular interest is a general proof that in an arbitrary homogeneous chamber the beam-size corrections vanish for any axisymmetric beam current distribution.
\end{abstract}

DOI: 10.1103/PhysRevSTAB.4.092801

PACS numbers: 41.75.-i, 41.20.-q

\section{INTRODUCTION}

In many accelerators, especially in ion linacs and storage rings, beams occupy a significant fraction of the vacuum chamber cross section. On the other hand, an analysis of beam-induced signals in beam position monitors (BPMs) is usually restricted to the case of an infinitely small beam cross section (pencil beam). In this paper we consider the problem for a vacuum chamber with an arbitrary but constant cross section and calculate, for a given transverse charge distribution of an off-axis relativistic beam, the fields produced by the beam on the chamber wall. Comparing these fields with the fields of a pencil beam gives us corrections (e.g., to BPM signals) due to a finite transverse size of the beam.

Let a vacuum chamber have an arbitrary singleconnected cross section $S$ that does not change as a beam moves along the chamber axis $z$, and perfectly conducting walls. We consider the case of $(\omega b / \beta \gamma c)^{2} \ll 1$, where $\omega$ is the frequency of interest, $\beta c$ is the beam velocity, $\gamma=1 / \sqrt{1-\beta^{2}}$, and $b$ is a typical transverse dimension of the vacuum chamber. It includes both the ultrarelativistic limit, $\gamma \gg 1$, and the long-wavelength limit when, for

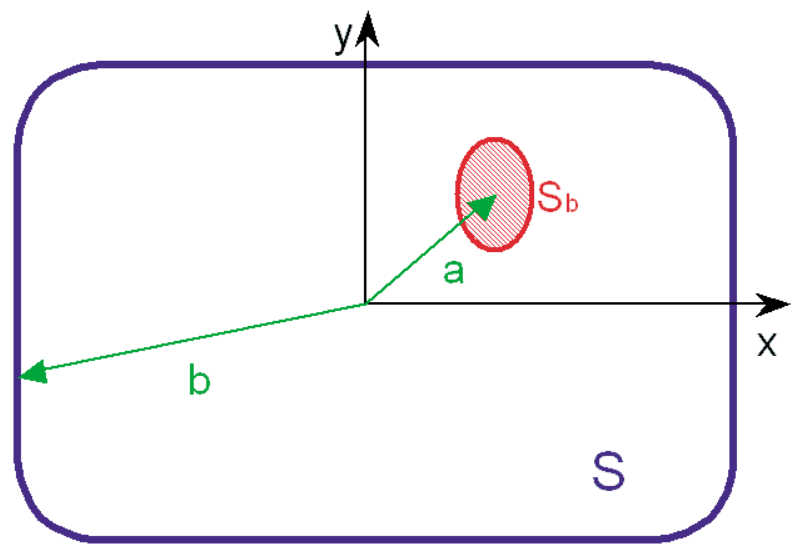

FIG. 1. (Color) Transverse cross section of the vacuum chamber $S$ and of the beam $S_{b}$.

$1098-4402 / 01 / 4(9) / 092801(8) \$ 15.00$ a fixed $\gamma$, the wavelength of interest $\lambda \gg 2 \pi b / \gamma$. Under these assumptions, the problem of calculating the beam fields at the chamber walls is reduced to a $2 \mathrm{D}$ electrostatic problem of finding the field of the transverse distribution $\lambda(\vec{r})$ of the beam charge, which occupies region $S_{b}$ of the beam cross section on the boundary $\partial S$ of region $S$ (see, e.g., [1]). The layout of the problem is illustrated in Fig. 1.

Let the beam charge distribution $\lambda(\vec{r})$ satisfy the normalization condition $\int_{S_{b}} d \vec{r} \lambda(\vec{r})=1$, which means the unit charge per unit length of the bunch. If we know the field $e(\vec{r}, \vec{b})$ produced at a point $\vec{b}$ on the wall by a pencil beam located at a point $\vec{r}$ of region $S_{b}$, the field of the distribution is given by

$$
E(\vec{a}, \vec{b})=\int_{S_{b}} d \vec{r} \lambda(\vec{r}) e(\vec{r}, \vec{b}),
$$

where the vector $\vec{a}$ is defined as the center of the charge distribution: $\vec{a}=\int d \vec{r} \vec{r} \lambda(\vec{r})$. Obviously, the case of a pencil beam corresponds to the distribution $\lambda(\vec{r})=\delta(\vec{r}-\vec{a})$, where $\delta(\vec{r})$ is the 2D $\delta$ function. Let us start from a particular case of a circular cylindrical vacuum chamber.

\section{CIRCULAR CHAMBER}

In a circular cylindrical pipe of radius $b$, a pencil beam with a transverse offset $\vec{r}$ from the axis produces the following electric field on the wall:

$$
\begin{aligned}
e(\vec{r}, \vec{b}) & =\frac{1}{2 \pi b} \frac{b^{2}-r^{2}}{b^{2}-2 b r \cos (\theta-\varphi)+r^{2}} \\
& =\frac{1}{2 \pi b}\left\{1+2 \sum_{k=1}^{\infty}\left(\frac{r}{b}\right)^{k} \cos [k(\theta-\varphi)]\right\},
\end{aligned}
$$

where $\varphi, \theta$ are the azimuthal angles of vectors $\vec{r}, \vec{b}$, correspondingly. One should note that this field is normalized as follows:

$$
\oint_{\partial S} d l e(\vec{r}, \vec{b})=1
$$

(C) 2001 The American Physical Society

092801-1 
where integration goes along the boundary $\partial S$ of the transverse cross section of the vacuum chamber.

Integrating the multipole expansion in the right-hand side (rhs) of Eq. (2) with a double-Gaussian distribution of the beam charge

$$
\lambda(x, y)=\frac{1}{2 \pi \sigma_{x} \sigma_{y}} \exp \left[-\frac{\left(x-a_{x}\right)^{2}}{2 \sigma_{x}^{2}}-\frac{\left(y-a_{y}\right)^{2}}{2 \sigma_{y}^{2}}\right],
$$

assuming, of course, that the rms beam sizes are small, $\sigma_{x}, \sigma_{y} \ll b$, one obtains nonlinearities in the form of powers of $a_{x}, a_{y}$, as well as the beam size corrections, which come as powers of $\sigma_{x}, \sigma_{y}$. To our knowledge, this was done first for the double-Gaussian beam in a circular pipe by Miller et al. [2], where the expansion was cal- culated up to the third order terms. More recently, their results have been used at LANL in measuring secondorder beam moments with BPMs and calculating the beam emittance from the measurements [3]. In a recent series of papers [4] by CERN authors, the results [2] have been recalculated (and corrected in the third order) and used to derive the beam size from measurements with movable BPMs.

In fact, integrating (2) with the distribution (3) can be readily carried out up to an arbitrary order. Using in Eq. (2) the binomial expansion for

$$
r^{k} \cos [k(\theta-\varphi)]=\operatorname{Re}\left[e^{i k \theta}(x-i y)^{k}\right]
$$

makes the $x$ and $y$ integrations very simple, and the $k$ th order term ( $k$-pole) of the resulting expansion is

$$
E^{(k)}(\theta)=\frac{k !}{\pi b} \sum_{m=0}^{k} \cos \left(\frac{m \pi}{2}-k \theta\right) \frac{x_{0}^{k-m} y_{0}^{m}}{b^{k}} \sum_{s=0}^{[(k-m) / 2]} \frac{\left(\sigma_{x}^{2} / 2 x_{0}^{2}\right)^{s}}{s !(k-m-2 s) !} \sum_{p=0}^{[m / 2]} \frac{\left(\sigma_{y}^{2} / 2 y_{0}^{2}\right)^{p}}{p !(m-p) !},
$$

where $x_{0}, y_{0}$ stand for the beam center coordinates $a_{x}, a_{y}$. Explicitly, up to the fifth order terms,

$$
\begin{aligned}
E\left(\vec{r}_{0}, \vec{b}\right)= & \frac{1}{2 \pi b}+\frac{1}{\pi b^{2}}\left\{\cos \theta x_{0}+\sin \theta y_{0}\right\}+\frac{1}{\pi b^{3}}\left\{\cos 2 \theta\left(\sigma_{x}^{2}-\sigma_{y}^{2}+x_{0}^{2}-y_{0}^{2}\right)+\sin 2 \theta 2 x_{0} y_{0}\right\} \\
& +\frac{1}{\pi b^{4}}\left\{\cos 3 \theta x_{0}\left[3\left(\sigma_{x}^{2}-\sigma_{y}^{2}\right)+x_{0}^{2}-3 y_{0}^{2}\right]+\sin 3 \theta y_{0}\left[3\left(\sigma_{x}^{2}-\sigma_{y}^{2}\right)+3 x_{0}^{2}-y_{0}^{2}\right]\right\} \\
& +\frac{1}{\pi b^{5}}\left\{\cos 4 \theta\left[3\left(\sigma_{x}^{2}-\sigma_{y}^{2}+x_{0}^{2}-y_{0}^{2}\right)^{2}-2 x_{0}^{4}-2 y_{0}^{4}\right]+\sin 4 \theta 4 x_{0} y_{0}\left[3\left(\sigma_{x}^{2}-\sigma_{y}^{2}\right)+x_{0}^{2}-y_{0}^{2}\right]\right\} \\
& +\frac{1}{\pi b^{6}}\left\{\cos 5 \theta x_{0}\left[15\left(\sigma_{x}^{2}-\sigma_{y}^{2}\right)^{2}+10\left(\sigma_{x}^{2}-\sigma_{y}^{2}\right)\left(x_{0}^{2}-3 y_{0}^{2}\right)+x_{0}^{4}-10 x_{0}^{2} y_{0}^{2}+5 y_{0}^{4}\right]\right. \\
& \left.\quad+\sin 5 \theta y_{0}\left[15\left(\sigma_{x}^{2}-\sigma_{y}^{2}\right)^{2}+10\left(\sigma_{x}^{2}-\sigma_{y}^{2}\right)\left(3 x_{0}^{2}-y_{0}^{2}\right)+5 x_{0}^{4}-10 x_{0}^{2} y_{0}^{2}+y_{0}^{4}\right]\right\}+\cdots
\end{aligned}
$$

The multipole expansion (5) that includes terms up to the decapoles leads us to an interesting observation: All beam-size corrections come in the form of the difference $\sigma_{x}^{2}-\sigma_{y}^{2}$ and vanish for a round beam where $\sigma_{x}^{2}=\sigma_{y}^{2}$. This would be obvious for an on-axis beam in a round pipe from the Gauss law, but for a deflected beam the result seems rather remarkable.

It is not easy to see directly from Eq. (4) whether the beam-size corrections for a round beam in a round pipe vanish in all orders. However, one can check explicitly that this is the case. Let us consider an arbitrary azimuthally symmetric distribution of the beam charge $\tilde{\lambda}(\vec{r})=\tilde{\lambda}(r)$, where the tilde in $\tilde{\lambda}$ means that the argument of the distribution function $\lambda$ is shifted so that the vector $\vec{r}$ now originates from the beam center: $\lambda(\vec{a}+\vec{r})=\tilde{\lambda}(\vec{r})$.

In this case, the integration in Eq. (1) for the circular pipe can be done explicitly. Namely, using the expansion in Eq. (2) and integrating in polar coordinates $(r, \varphi)$, for the case when $\tilde{\lambda}(r, \varphi)=\tilde{\lambda}(r)$ one can write

$$
\begin{aligned}
E(\theta) & =\frac{1}{2 \pi b} \int_{0}^{\infty} r d r \tilde{\lambda}(r) \int_{0}^{2 \pi} \frac{d \varphi\left(b^{2}-a^{2}-r^{2}-2 b r \cos \varphi\right)}{b^{2}+a^{2}+r^{2}-2 a b \cos \theta+2 a r \cos \varphi-2 b r \cos (\varphi-\theta)} \\
& =\frac{1}{2 \pi b} \int_{0}^{\infty} 2 \pi r d r \tilde{\lambda}(r) \frac{b^{2}-a^{2}}{b^{2}+a^{2}-2 a b \cos \theta} \\
& =\frac{1}{2 \pi b} \frac{b^{2}-a^{2}}{b^{2}+a^{2}-2 a b \cos \theta} .
\end{aligned}
$$

The last expression follows from the preceding line due to the charge normalization, and it is exactly the field of a pencil beam displaced from the chamber axis by $\vec{a}=(a, 0)$; compare Eq. (2). The only real effort here was to perform 


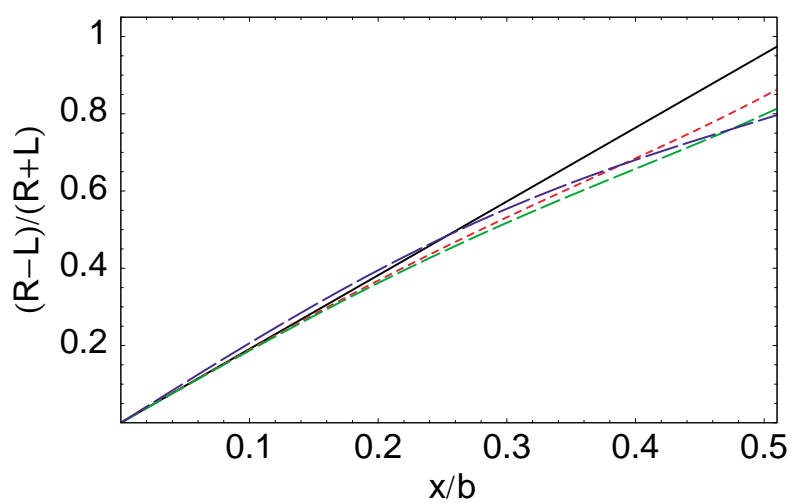

FIG. 2. (Color) Beam position monitor (BPM) signal ratio (7) in a circular chamber versus beam center position $x / b$ for three vertical beam offsets $y / b=0,1 / 4,1 / 2$ (short-dashed line, dashed line, long-dashed line, respectively) without beam-size corrections (pencil beam, $\sigma_{x}=\sigma_{y}=0$ ). The solid line shows the linear part of the BPM response.

the angular integration, which turns out to be independent of $r$. It was done analytically by introducing a new complex variable $z, \cos \varphi=\left(z+z^{-1}\right) / 2$, and then integrating along a unit circle in the complex $z$ plane with residues. ${ }^{1}$

We now apply the above results for calculating signals in a BPM. First, we assume that signals induced in BPM electrodes (strip lines or buttons) are proportional to the wall image current integrated within the transverse extent of the electrode on the chamber wall. Such an assumption is usually made in analytical treatments of BPM signals (see, e.g., $[1,2,4,5]$ ) and is justified when the BPM electrodes are flush with the chamber walls, grounded, and separated from the wall by narrow gaps. Certainly, there are some field distortions due to the presence of the gaps, but they are rather small for narrow gaps. Moreover, even

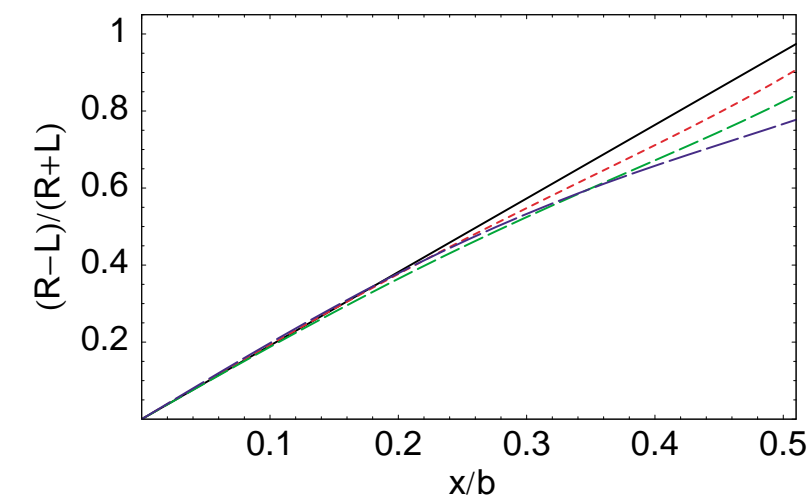

FIG. 3. (Color) Same as Fig. 2, but with $\sigma_{x} / b=0.2, \sigma_{y}=0$.

\footnotetext{
${ }^{1}$ Trying to perform this integration with MATHEMATICA, I found a bug in its analytical integration package for this particular kind of integral. Wolfram Research acknowledged the bug, and they are working to fix it.
}

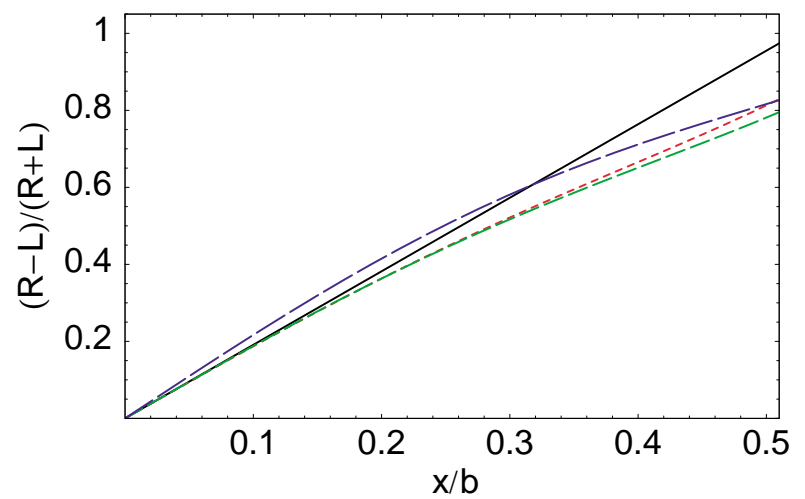

FIG. 4. (Color) Same as Fig. 2, but with $\sigma_{x}=0, \sigma_{y} / b=0.2$.

for a more complicated BPM geometry with realistic strip lines protruding inside a circular pipe, it was demonstrated by measurements (see [5]) and by numerical 3D modeling [6] that the effects of field distortions near the strip line edges can be accounted for by integrating the wall current within an effective transverse extent of the strip lines (slightly larger than their width) in a simple smooth-pipe model with the effective pipe radius taken to be an average of the strip line inner radius and the inner radius of the beam pipe.

Consider now in a circular chamber of radius $b$ a strip line BPM with a pair of electrodes in the horizontal plane. Let us assume that the strip line electrodes are flush with the chamber walls, grounded, and have subtended angle $\phi$ per strip line. Following the discussion above, we neglect the field distortions near the strip edges and calculate the signals induced on the strip line electrodes by integrating the field (5) over the interval $-\phi / 2 \leq \theta \leq \phi / 2$ for the right electrode $(R)$ and over $\pi-\phi / 2 \leq \theta \leq \pi+\phi / 2$ for the left electrode $(L)$. The ratio of the difference between the signals on the right and left electrodes in the horizontal plane to the sum of these signals is

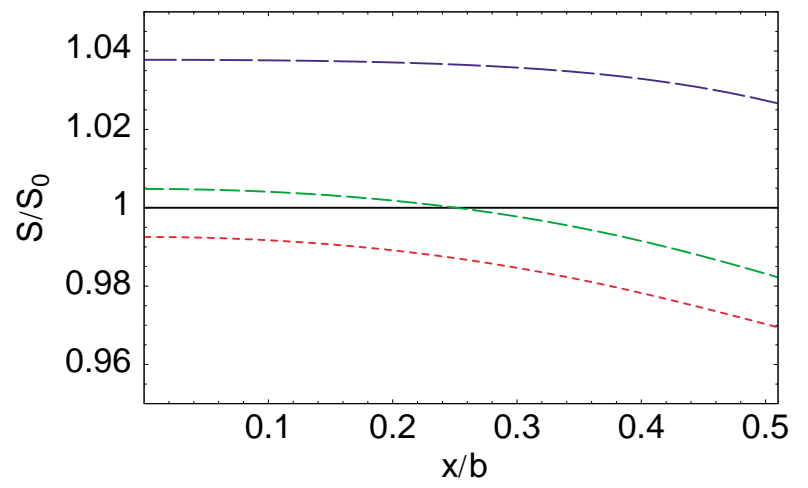

FIG. 5. (Color) Relative magnitude of beam-size corrections in a circular chamber with $\sigma_{x} / b=0.1, \sigma_{y} / b=0.2$ for three vertical beam offsets $y / b=0,1 / 4,1 / 2$ (short-dashed line, dashed line, long-dashed line, respectively). Here " 1 " corresponds to a pencil beam case, i.e., to one of the three curves in Fig. 2 for the corresponding beam vertical offset. 


$$
\begin{aligned}
\frac{R-L}{R+L}=2 \frac{x_{0}}{b} \frac{\sin \phi / 2}{\phi / 2}\{ & 1-\frac{2}{b^{2}} \frac{\sin \phi}{\phi}\left(\sigma_{x}^{2}-\sigma_{y}^{2}+x_{0}^{2}-y_{0}^{2}\right)+\frac{1}{b^{2}} \frac{\sin 3 \phi / 2}{3 \phi / 2}\left(\sigma_{x}^{2}-\sigma_{y}^{2}+x_{0}^{2} / 3-y_{0}^{2}\right) \\
& -\frac{2}{b^{4}} \frac{\sin 2 \phi}{2 \phi}\left[\left(\sigma_{x}^{2}-\sigma_{y}^{2}+x_{0}^{2}-y_{0}^{2}\right)^{2}-2 x_{0}^{4}-2 y_{0}^{4}\right]+\frac{4}{b^{4}}\left(\frac{\sin \phi}{\phi}\right)^{2}\left(\sigma_{x}^{2}-\sigma_{y}^{2}+x_{0}^{2}-y_{0}^{2}\right)^{2} \\
& \left.+\frac{1}{b^{4}} \frac{\sin 5 \phi / 2}{5 \phi / 2}\left[3\left(\sigma_{x}^{2}-\sigma_{y}^{2}\right)^{2}+2\left(\sigma_{x}^{2}-\sigma_{y}^{2}\right)\left(x_{0}^{2}-3 y_{0}^{2}\right)+x_{0}^{4} / 5-2 x_{0}^{2} y_{0}^{2}+y_{0}^{4}\right]+O\left(b^{-6}\right)\right\} .
\end{aligned}
$$

The factor outside of the brackets in the rhs of Eq. (7) is the linear part of the BPM response, so that all terms in the brackets except one are nonlinearities and beam-size corrections.

Corrections (7) are shown in Figs. $2-5$ for a $60^{\circ}$ strip line BPM. Figure 2 shows the nonlinearities of the BPM response for a pencil beam. The signal ratio changes are noticeable when Figs. 3 and 4 for flat beams are compared with Fig. 2. In Fig. 5, the ratio $S / S_{0}$ is plotted versus the beam center position. Here $S$ is the difference-over-sum ratio, $(R-L) /(R+L)$, for a finte-size beam, and $S_{0}$ is the same ratio for a pencil beam. One can see from Fig. 5 that, for a reasonable transverse beam size, the beam-size corrections are on the level of a few percent.

\section{VACUUM CHAMBER OF ARBITRARY CROSS SECTION}

Let us consider now a more general case of a homogeneous vacuum chamber with an arbitrary single-connected cross section $S$. The field $e(\vec{r}, \vec{b})$ produced by a pencil beam at a point $\vec{b}$ on the wall can be written as (see, e.g., $[7,8])$

$$
e(\vec{r}, \vec{b})=-\sum_{s} k_{s}^{-2} e_{s}(\vec{r}) \nabla_{\nu} e_{s}(\vec{b}),
$$

where $s=(n, m)$ is a generalized (2D) index, $\nabla_{\nu}=\vec{\nabla} \cdot \overrightarrow{\hat{\nu}}$ is a normal derivative at the point $\vec{b}$ on the region boundary $\partial S(\vec{\nabla}$ is the $2 \mathrm{D}$ gradient operator, $\overrightarrow{\hat{\nu}}$ means an outward normal vector to the boundary), and $k_{s}^{2}, e_{s}(\vec{r})$ are eigenvalues and orthonormalized eigenfunctions of the following 2D Dirichlet problem in the region $S$ :

$$
\left(\nabla^{2}+k_{s}^{2}\right) e_{s}(\vec{r})=0 ; \quad e_{s}(\vec{r} \in \partial S)=0 .
$$

The expansion (8) follows from the fact that

$$
\Phi(\vec{r}-\vec{a})=\sum_{s} k_{s}^{-2} e_{s}(\vec{r}) e_{s}(\vec{a})
$$

is the Green function of the problem (9), which means that it satisfies the equation

$$
\nabla^{2} \Phi(\vec{r}-\vec{a})=-\delta(\vec{r}-\vec{a}) .
$$

In other words, $\Phi(\vec{r}-\vec{a})$ is (up to a factor $1 / \varepsilon_{0}$ ) an electric potential created at point $\vec{r}$ of region $S$ by a unit point charge placed at point $\vec{a}$. One can easily check that substituting the sum (10) into Eq. (11) gives, with the account of (9), the correct result due to the following property of eigenfunctions:

$$
\sum_{s} e_{s}(\vec{r}) e_{s}(\vec{a})=\delta(\vec{r}-\vec{a})
$$

The eigenfunctions for simple regions such as a circle or a rectangle can be found in electrodynamics textbooks (see also the Appendix in Ref. [7]). For the circular case, summing the corresponding Bessel functions in (8) leads directly to the last expression in Eq. (2).

For a thick beam with a given transverse charge distribution, one can write from Eqs. (1) and (8)

$$
E(\vec{a}, \vec{b})=-\sum_{s} k_{s}^{-2} \nabla_{\nu} e_{s}(\vec{b}) \int_{S_{b}} \vec{\lambda}(\vec{r}) e_{s}(\vec{a}+\vec{r}) d \vec{r}
$$

where again the tilde in $\tilde{\lambda}$ means an argument shift in the distribution function $\lambda, \lambda(\vec{a}+\vec{r})=\tilde{\lambda}(\vec{r})$, so that the integration vector $\vec{r}$ originates from the beam center $\vec{a}$. Performing the Taylor expansion of the eigenfunction $e_{s}(\vec{a}+\vec{r})$ around point $\vec{a}$,

$$
\begin{aligned}
e_{s}(\vec{a}+\vec{r}) & =\sum_{m=0}^{\infty}(\vec{r} \vec{\nabla})^{m} e_{s}(\vec{a}) / m ! \\
& =e_{s}(\vec{a})+\vec{r} \vec{\nabla} e_{s}(\vec{a})+\frac{1}{2}(\vec{r} \vec{\nabla})^{2} e_{s}(\vec{a})+\cdots,
\end{aligned}
$$

and integrating in (13) leads to the following multipole series:

$$
E(\vec{a}, \vec{b})=-\sum_{s} k_{s}^{-2} \nabla_{\nu} e_{s}(\vec{b}) \sum_{m=0}^{\infty} \sum_{i_{1}=1}^{2} \sum_{i_{2}=1}^{2} \cdots \sum_{i_{m}=1}^{2} \partial_{i_{1}} \partial_{i_{2}} \cdots \partial_{i_{m}} e_{s}(\vec{a}) / m ! \int_{S_{b}} d \vec{r} \tilde{\lambda}(\vec{r}) r_{i_{1}} r_{i_{2}} \cdots r_{i_{m}},
$$

where $\partial_{i} \equiv \partial / \partial r_{i}, i=1,2$, and all effects of the finite beam size here enter through the components of the multipoles of the beam charge distribution.

If we restrict ourselves by considering only symmetric (with respect to two axis) charge distributions, i.e., assume $\tilde{\lambda}(-\vec{r})=\tilde{\lambda}(\vec{r})$, all integrals for odd $m$ in (14) vanish, and the general expansion (14) can be significantly simplified: 


$$
\begin{aligned}
E(\vec{a}, \vec{b}) & =e(\vec{a}, \vec{b})+\frac{1}{2} \partial_{x}^{2} e(\vec{a}, \vec{b}) \int_{S_{b}} d \vec{r} \tilde{\lambda}(\vec{r})\left(x^{2}-y^{2}\right)+\frac{1}{24} \partial_{x}^{4} e(\vec{a}, \vec{b}) \int_{S_{b}} d \vec{r} \tilde{\lambda}(\vec{r})\left(x^{4}-6 x^{2} y^{2}+y^{4}\right)+\cdots \\
& =e(\vec{a}, \vec{b})+\sum_{n=1}^{\infty} \partial_{x}^{2 n} e(\vec{a}, \vec{b}) M_{2 n} /(2 n) !
\end{aligned}
$$

In obtaining the last expression, the following property of the sum (14) was used: Flipping the derivatives, $\partial_{y}^{2} e_{s}(\vec{a})=-\partial_{x}^{2} e_{s}(\vec{a})$, inside the sum does not change the result. This is due to $\partial_{y}^{2} e_{s}(\vec{a})=-\left(\partial_{x}^{2}+k_{s}^{2}\right) e_{s}(\vec{a})$ from Eq. (9), and because any extra factor $k_{s}^{2}$ in (14) leads to a zero sum since it just gives a derivative of the $\delta$ function [cf. (12)] with a nonzero argument because of $\vec{a} \neq \vec{b}$ (the beam does not touch the wall).

Equation (15) is more transparent than (14). Let us take a look at the moments in (15) in their closed form:

$$
\begin{aligned}
M_{2 n}= & \int_{S_{b}} d \vec{r} \tilde{\lambda}(\vec{r}) \\
& \times\left[x^{2 n}-C_{2}^{2 n-2} x^{2 n-2} y^{2}+C_{4}^{2 n-4} x^{2 n-4} y^{4}\right. \\
& \left.\quad-\cdots+(-1)^{n} y^{2 n}\right]
\end{aligned}
$$

where $C_{k}^{n}=n ! /[k !(n-k) !]$ are binomial coefficients. It is useful to notice that the sum inside the square brackets in (16) is simply $\operatorname{Re}\left[(x+i y)^{2 n}\right]$, and in the polar coordinates of the beam Eq. (16) can be rewritten simply as

$$
M_{2 n}=\int_{S_{b}} d \vec{r} \tilde{\lambda}(\vec{r}) r^{2 n} \cos 2 n \varphi .
$$

Now it is quite obvious that, if one assumes an arbitrary azimuthally-symmetric distribution of the beam charge $\tilde{\lambda}(\vec{r})=\tilde{\lambda}(r)$, i.e., $\tilde{\lambda}(r, \varphi)=\tilde{\lambda}(r)$, all beam moments (17) become equal to zero after the angular integration and the corresponding beam-size corrections in (15) vanish. Therefore, we proved a rather general statement: The fields produced by an ultrarelativistic beam with an azimuthally-symmetric charge distribution on the walls of a homogeneous vacuum chamber of an arbitrary cross section are exactly the same as those due to a pencil beam of the same current following the same path. A particular case of this statement, for a circular chamber cross section, was proved by explicit calculations earlier (see Sec. II).

The physical explanation of this effect is simple. The electric field outside the beam $\vec{E}$ is a superposition of the field due to the charge distribution itself, $\vec{E}_{\mathrm{vac}}^{\mathrm{dis}}$, and the field due to induced charges on the chamber walls, $\vec{E}_{\text {ind }}$. From the Gauss law, for an azimuthally-symmetric beam charge distribution, the field $\vec{E}_{\text {vac }}^{\text {dis }}$ outside the beam (in vacuum, without the chamber) is exactly the same as that of a pencil beam, $\vec{E}_{\mathrm{vac}}^{0}$, if the last one has the same charge and travels along the axis of the thick beam. Therefore, the induced charge distribution on the wall will be identical for the thick and pencil beams, and as a result the same will be true for the total electric field outside the beam [9].

The expansion (15) for symmetric distributions of the beam charge gives the beam-size corrections for an arbitrary chamber, as long as the beam charge distribution is known. As two particular symmetric charge distributions of practical interest, we consider a double-Gaussian beam [cf. Eq. (3)],

$$
\tilde{\lambda}(x, y)=\exp \left(-x^{2} / 2 \sigma_{x}^{2}-y^{2} / 2 \sigma_{y}^{2}\right) /\left(2 \pi \sigma_{x} \sigma_{y}\right),
$$

and a uniform beam with a rectangular cross section $2 \sigma_{x} \times 2 \sigma_{y}$,

$$
\tilde{\lambda}(x, y)=\frac{\theta\left(x+\sigma_{x}\right) \theta\left(\sigma_{x}-x\right) \theta\left(y+\sigma_{y}\right) \theta\left(\sigma_{y}-y\right)}{4 \sigma_{x} \sigma_{y}},
$$

where $\theta(x)$ is the step function. The two distributions $\tilde{\lambda}$ above are written in the beam coordinates, with $x=$ $y=0$ corresponding to the beam center, as discussed after Eq. (5).

For the double-Gaussian beam (18), $M_{2}=\sigma_{x}^{2}-\sigma_{y}^{2}$, $M_{4}=3\left(\sigma_{x}^{2}-\sigma_{y}^{2}\right)^{2}$, etc., so that Eq. (15) takes the form

$$
\begin{aligned}
E(\vec{a}, \vec{b})= & e(\vec{a}, \vec{b})+\frac{1}{2}\left(\sigma_{x}^{2}-\sigma_{y}^{2}\right) \partial_{x}^{2} e(\vec{a}, \vec{b}) \\
& +\frac{1}{8}\left(\sigma_{x}^{2}-\sigma_{y}^{2}\right)^{2} \partial_{x}^{4} e(\vec{a}, \vec{b}) \\
& +\frac{1}{48}\left(\sigma_{x}^{2}-\sigma_{y}^{2}\right)^{3} \partial_{x}^{6} e(\vec{a}, \vec{b})+O\left(\sigma^{8}\right) .
\end{aligned}
$$

Similarly, for the uniform beam with the rectangular cross section (19), the corrections are

$$
\begin{aligned}
E(\vec{a}, \vec{b})= & e(\vec{a}, \vec{b})+\frac{1}{6}\left(\sigma_{x}^{2}-\sigma_{y}^{2}\right) \partial_{x}^{2} e(\vec{a}, \vec{b})+\frac{1}{40}\left(\sigma_{x}^{4}-\frac{10}{3} \sigma_{x}^{2} \sigma_{y}^{2}+\sigma_{y}^{4}\right) \partial_{x}^{4} e(\vec{a}, \vec{b}) \\
& +\frac{1}{5040}\left(\sigma_{x}^{6}-7 \sigma_{x}^{4} \sigma_{y}^{2}+7 \sigma_{x}^{2} \sigma_{y}^{4}-\sigma_{y}^{6}\right) \partial_{x}^{6} e(\vec{a}, \vec{b})+O\left(\sigma^{8}\right) .
\end{aligned}
$$

One can see that for a round beam, $\sigma_{x}=\sigma_{y}$, all corrections in (20) disappear as expected, and for a square beam cross section in (20) the lowest correction is proportional to $\sigma^{4}$, while the next-order one is proportional to $\sigma^{8}$.

One should note at this point that the general field expansion (15) and Eqs. (20) and (21) derived above are essentially 
the expansions in a small parameter $\sigma^{2} / b^{2}$, where $\sigma$ is a typical transverse beam size and $b$ stands for a characteristic transverse dimension of the chamber cross section. The powers of $1 / b$ are produced by the derivatives of the pencil beam field $e(\vec{a}, \vec{b})$ in Eqs. (15), (20), and (21). Therefore, these results are valid for any beam offset $a$, large or small, no matter what the relation is between $\sigma$ and $a$.
Equations (15), (20), and (21) give us a rather good idea about how the beam-size corrections enter into the field expressions. The nonlinearities, however, are hidden in the pencil-beam field $e(\vec{a}, \vec{b})$ and in its derivatives. We can single out the nonlinearities in a manner similar to the one used to obtain the beam-size corrections, by expanding the field $e(\vec{a}, \vec{b})$ in powers of $a$ around the chamber axis:

$$
e(\vec{a}, \vec{b})=\sum_{m=0}^{\infty}(\vec{a} \vec{\nabla})^{m} e(0, \vec{b}) / m !=e_{0}+\vec{a} \vec{\nabla} e_{0}+\frac{1}{2}(\vec{a} \vec{\nabla})^{2} e_{0}+\cdots
$$

where the notation $e_{0}=e(0, \vec{b})$ was introduced for brevity, and similarly for the derivatives. In the most general case, unfortunately, it does not lead to convenient equations. However, for vacuum chambers with some symmetry the results can be simplified significantly. Here we limit our consideration to the case of region $S$ that is symmetric with respect to its vertical and horizontal axes. If a pair of narrow BPM electrodes is placed on the walls in the horizontal plane of such a two-axis symmetric chamber, the fields due to a pencil beam at location $\vec{a}=\left(x_{0}, y_{0}\right)$ on the right $(R)$ and left $(L)$ electrodes do not change when $y_{0} \leftrightarrow-y_{0}$ (i.e., they are even functions of $\left.y_{0}\right)$. Moreover, from the vertical symmetry, $L\left(x_{0}, y_{0}\right)=R\left(-x_{0}, y_{0}\right)$. Using these properties, as well as the same trick $\partial_{y}^{2} e_{s}=-\partial_{x}^{2} e_{s}$ as above in the sum for derivatives of $e_{0}$, we obtain the differenceover-sum signal ratio of BPM signals in a rather general form:

$$
\begin{aligned}
\frac{R-L}{R+L}=\frac{x_{0} \partial_{x} e_{0}}{e_{0}}\{ & 1+\frac{1}{2} \frac{\partial_{x}^{3} e_{0}}{\partial_{x} e_{0}}\left(\frac{x_{0}^{2}}{3}-y_{0}^{2}+M_{2}\right)-\frac{1}{2} \frac{\partial_{x}^{2} e_{0}}{e_{0}}\left(x_{0}^{2}-y_{0}^{2}+M_{2}\right) \\
& +\frac{1}{24} \frac{\partial_{x}^{5} e_{0}}{\partial_{x} e_{0}}\left[\frac{x_{0}^{4}}{5}-2 x_{0}^{2} y_{0}^{2}+y_{0}^{4}+2 M_{2}\left(x_{0}^{2}-3 y_{0}^{2}\right)+M_{4}\right] \\
& \left.-\frac{1}{24} \frac{\partial_{x}^{4} e_{0}}{e_{0}}\left[x_{0}^{4}-6 x_{0}^{2} y_{0}^{2}+y_{0}^{4}+6 M_{2}\left(x_{0}^{2}-y_{0}^{2}\right)+M_{4}\right]+O\left(r_{0}^{6} / b^{6}, \sigma^{6} / b^{6}\right)\right\},
\end{aligned}
$$

where the nonlinearities are shown explicitly as powers of $x_{0}$ and $y_{0}$, and all beam-size corrections enter via the even moments $M_{2 n}$ of the beam charge distribution [cf. Eq. (15)]. It still takes some effort to arrive at the particular case of Eq. (22) for the circular pipe, Eq. (7), but the structure of corrections is rather clear.

We conclude our study of the general case with a remark that the pencil beam field $e(\vec{a}, \vec{b})$ and its derivatives are generally not easy to calculate, except for a few particular cases. For a circular pipe, we know the explicit expression (2) for $e(\vec{a}, \vec{b})$. Another case where the eigenfunctions are simple and the sums in Eqs. (15) and (22) can be worked out relatively easily is a rectangular chamber.

\section{RECTANGULAR CHAMBER}

Let us consider a vacuum chamber with the cross section $S$ having a rectangular shape with width $w$ and height $h$. The orthonormalized eigenfunctions of the boundary problem (9) for region $S$ are

$$
e_{n, m}(x, y)=\frac{2}{\sqrt{w h}} \sin \pi n\left(\frac{1}{2}+\frac{x}{w}\right) \sin \pi m\left(\frac{1}{2}+\frac{y}{h}\right),
$$

where $-w / 2 \leq x \leq w / 2, \quad-h / 2 \leq y \leq h / 2$, and $n, m=1,2, \ldots$ Summing up in Eq. (8) for this case gives us the field produced by a pencil beam,

$$
\begin{aligned}
e\left(\vec{r}_{0}, \vec{b}\right)= & \sum_{m=1}^{\infty} \sin \pi m\left(\frac{h+y_{0}}{2 h}\right) \sin \pi m\left(\frac{h+y_{h}}{2 h}\right) \\
& \times \frac{2 \sinh \pi m\left[\left(w / 2+x_{0}\right) / h\right]}{h \sinh (\pi m w / h)},
\end{aligned}
$$

at point $\vec{b}=\left(w / 2, y_{h}\right)$ on the right side wall. Should we consider a left wall point instead, $\vec{b}=\left(-w / 2, y_{h}\right)$, the only change in (23) would be the replacement $x \rightarrow-x$ (see Sec. III for more general consideration of the symmetry). For points on the top or bottom walls, one should exchange $w \leftrightarrow h, x \leftrightarrow y$, and $y_{h} \leftrightarrow x_{w}$ in Eq. (23). Unlike the circular pipe case, we are still left with a sum in Eq. (23), but the series is fast (exponentially) converging and convenient for calculations; e.g., see $[7,8]$. In particular, it is very easy here to calculate derivatives required in Eqs. (15) and (20) $-(22) ; \partial_{x}^{2} e(\vec{r}, \vec{b})$ is given by the same series (23), only with an extra factor $(\pi m / h)^{2}$ in the sum. In fact, for the particular charge distributions (18) and (19) considered above, it is simple enough to perform the integration (1) directly using (23), which produces 


$$
E\left(\vec{r}_{0}, \vec{b}\right)=\sum_{m=1}^{\infty} \sin \pi m\left(\frac{h+y_{0}}{2 h}\right) \sin \pi m\left(\frac{h+y_{h}}{2 h}\right) \frac{2 \sinh \pi m\left(\frac{w}{2 h}+\frac{x_{0}}{h}\right)}{h \sinh (\pi m w / h)} f\left(\frac{\pi m \sigma_{y}}{h}\right) F\left(\frac{\pi m \sigma_{x}}{w}\right) .
$$

The beam-size corrections in (24) enter as the form factors $f(z), F(z)$. For the double-Gaussian charge distribution (18), the form factors are $f(z)=\exp \left(-z^{2} / 2\right), F(z)=\exp \left(z^{2} / 2\right)$, so that the correction factor in (24) takes the form

$$
f\left(\frac{\pi m \sigma_{y}}{h}\right) F\left(\frac{\pi m \sigma_{x}}{w}\right)=\exp \left[\left(\frac{\pi m}{h}\right)^{2} \frac{\sigma_{x}^{2}-\sigma_{y}^{2}}{2}\right] .
$$

Obviously, for an axisymmetric beam with $\sigma_{x}=\sigma_{y}$, the argument of the exponent vanishes, and the exponent is equal to unity. As a result, the field (24) of a finite-size axisymmetric beam will be exactly equal to that of a pencil beam, Eq. (23).

For the uniform rectangular distribution (19), the form factors are $f(z)=\sin (z) / z, F(z)=\sinh (z) / z$, and the resulting correction factor is

$$
f\left(\frac{\pi m \sigma_{y}}{h}\right) F\left(\frac{\pi m \sigma_{x}}{w}\right)=\frac{\sin \left(\pi m \sigma_{y} / h\right)}{\pi m \sigma_{y} / h} \frac{\sinh \left(\pi m \sigma_{x} / h\right)}{\pi m \sigma_{x} / h} .
$$

Expanding this expression in powers of $\sigma$ leads to the conclusion that the lowest beam-size corrections here have the order of $\sigma^{4}$, as we already know from Sec. III.

As for BPM signals, the simplest way is to use the general result (22). For two strip line BPM electrodes of width $h_{1}$ on sidewalls of a rectangular vacuum chamber $w \times h$, the difference-over-sum signal ratio, up to the fifth order, is

$$
\begin{aligned}
\frac{R-L}{R+L}=\pi \frac{x_{0}}{h} \frac{\Sigma_{1}}{\Sigma_{0}}\{1 & +\frac{\pi^{2}}{2 h^{2}} \frac{\Sigma_{3}}{\Sigma_{1}}\left(\frac{x_{0}^{2}}{3}-y_{0}^{2}+M_{2}\right)-\frac{\pi^{2}}{2 h^{2}} \frac{\Sigma_{2}}{\Sigma_{0}}\left(x_{0}^{2}-y_{0}^{2}+M_{2}\right) \\
& +\frac{\pi^{4}}{24 h^{4}} \frac{\Sigma_{5}}{\Sigma_{1}}\left[\frac{x_{0}^{4}}{5}-2 x_{0}^{2} y_{0}^{2}+y_{0}^{4}+2 M_{2}\left(x_{0}^{2}-3 y_{0}^{2}\right)+M_{4}\right] \\
& \left.-\frac{\pi^{4}}{24 h^{4}} \frac{\Sigma_{4}}{\Sigma_{0}}\left[x_{0}^{4}-6 x_{0}^{2} y_{0}^{2}+y_{0}^{4}+6 M_{2}\left(x_{0}^{2}-y_{0}^{2}\right)+M_{4}\right]+O\left(r_{0}^{6} / b^{6}, \sigma^{6} / b^{6}\right)\right\},
\end{aligned}
$$

where $M_{2}, M_{4}$ are the moments of the beam charge distribution defined above, and

$$
\begin{aligned}
\Sigma_{2 n} & =\sum_{k=0}^{\infty}(2 k+1)^{2 n} \frac{\Phi\left[\pi(k+1 / 2) h_{1} / h\right]}{\cosh [\pi(k+1 / 2) w / h]}, \\
\Sigma_{2 n+1} & =\sum_{k=0}^{\infty}(2 k+1)^{2 n+1} \frac{\Phi\left[\pi(k+1 / 2) h_{1} / h\right]}{\sinh [\pi(k+1 / 2) w / h]},
\end{aligned}
$$

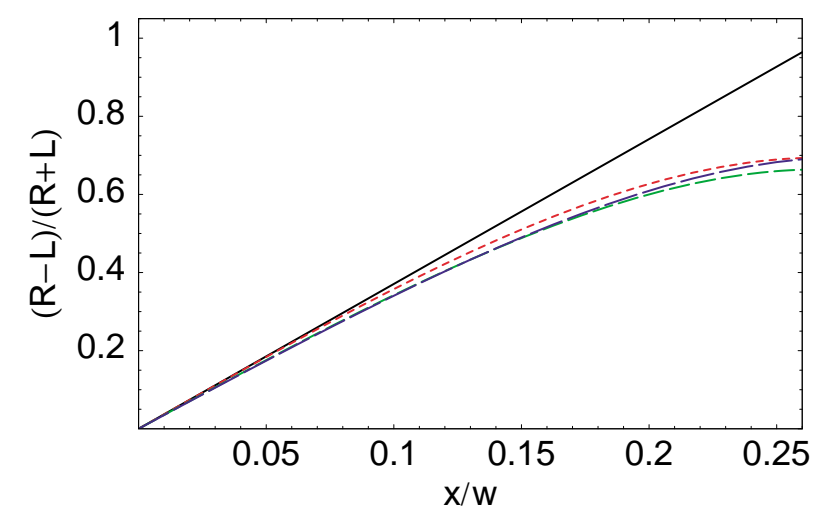

FIG. 6. (Color) BPM signal ratio (25) in a square chamber versus beam center position $x / w$ for three vertical beam offsets $y / h=0,1 / 8,1 / 4$ (short-dashed line, dashed line, long-dashed line, respectively) without beam-size corrections (pencil beam, $\left.\sigma_{x}=\sigma_{y}=0\right)$. The solid line shows the linear part of the BPM response. for $n=0,1,2, \ldots$ The sums above include one more form factor, $\Phi(z)=\sin z / z$, that accounts for the BPM electrode width. For narrow electrodes, when $h_{1} \ll h$, this form factor tends to 1 .

Corrections (25) are shown in Figs. 6-9 for a square chamber, $w=h$, and a BPM with very narrow electrodes, $h_{1}=h / 100$ (in fact, results for $h_{1}=h / 10$ are almost identical). Figure 6 shows only the nonlinearities, which in this case are practically the same for three different vertical beam offsets. On the contrary, the beam-size corrections here depend noticeably on the beam vertical offset, and range from about $+3 \%$ for $y=0$ (the chamber midplane)

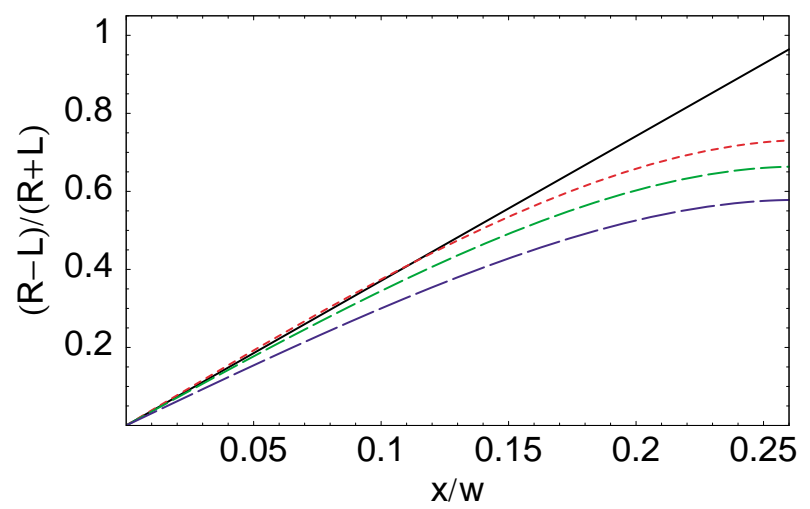

FIG. 7. (Color) Same as Fig. 6, but with $\sigma_{x} / w=0.1, \sigma_{y}=0$. 


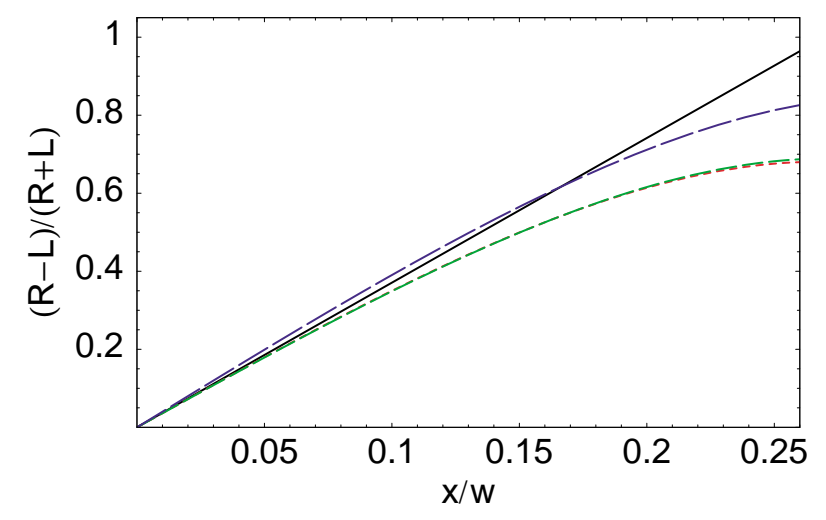

FIG. 8. (Color) Same as Fig. 6, but with $\sigma_{x}=0, \sigma_{y} / h=0.1$.

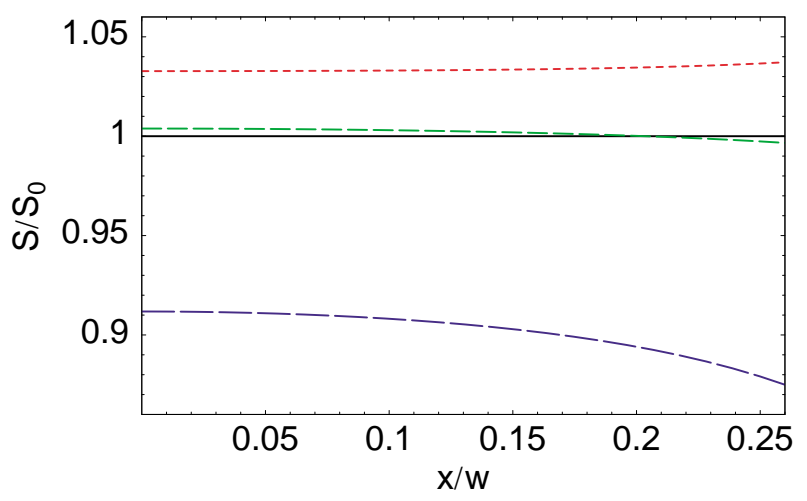

FIG. 9. (Color) Relative magnitude of beam-size corrections in a square chamber with $\sigma_{x} / w=0.1, \sigma_{y} / h=0.05$ for three vertical beam offsets $y / h=0,1 / 8,1 / 4$ (short-dashed line, dashed line, long-dashed line, respectively). Here " 1 " corresponds to a pencil beam case, i.e., to one of the three curves in Fig. 6 for the corresponding beam vertical offset.

to less than $1 \%$ for $y=h / 8$ to about $-(9-12) \%$ for $y=$ $h / 4$ (the beam is half way to the top wall), in the case of $\sigma_{x} / w=0.1, \sigma_{y} / h=0.05$ shown in Fig. 9. Similar to Fig. 5, in Fig. $9 S=(R-L) /(R+L)$ for a finite-size beam, and $S_{0}$ is the same ratio for a pencil beam, which is plotted in Fig. 6. Therefore, $S / S_{0}=1$ in Fig. 9 means that there are no corrections due to a finite transverse beam size. Again, as in the circular chamber, the beam-size corrections for flat beams can be rather significant, as we see from comparison of Figs. 7 and 8 with Fig. 6.

\section{CONCLUSIONS}

Nonlinearities and corrections due to a finite transverse beam size in beam fields and BPM signals are calculated for a homogeneous vacuum chamber in the case when the wavelength of interest is longer than a typical transverse dimension of the chamber and/or the beam is ultrarelativistic.

A general proof is presented that transverse beam-size corrections vanish in all orders for any azimuthally symmetric beam in an arbitrary chamber. One should emphasize that nonlinearities are still present in this case; for a given chamber cross section, they depend only on the displacement of the beam center from the chamber axis. However, the nonlinearities are the same for a finite-size axisymmetric beam and for a pencil beam (line source) with the same displacement. Having a nonsymmetric transverse distribution of the beam charge results in additional (properly beam-size) corrections. They tend to be minimal when the beam charge distribution is more symmetric.

Explicit analytical expressions are derived for two particular cases - circular and rectangular chamber cross section-as well as for the particular beam charge distributions - double-Gaussian and uniform rectangular distribution.

While we have not discussed this subject in the present paper, the calculated corrections to beam fields can be directly applied in calculating beam coupling impedances produced by small discontinuities of the vacuum chamber using the methods of Refs. [7,8].

\section{ACKNOWLEDGMENTS}

The author would like to acknowledge useful discussions with A. V. Aleksandrov and M. M. Blaskiewicz.

[1] J. H. Cuperus, Nucl. Instrum. Methods 145, 219 (1977).

[2] R. H. Miller et al., in Proceedings of the 12th International Conference on High-Energy Accelerators, Fermilab, 1983, edited by F. T. Cole and R. Donaldson (Fermilab, Batavia, IL, 1983), p. 602.

[3] S. J. Russel and B. E. Carlsten, in Proceedings of the 1999 Particle Accelerator Conference, New York (IEEE, Piscataway, NJ, 1999), p. 477.

[4] R. Assmann, B. Dehning, and J. Matheson, in Proceedings of the of 7th European Particle Accelerator Conference (EPAC2000), Vienna, 2000, http://accelconf.web.cern. ch/AccelConf/e00/index.html, p. 1693; ibid., p. 412; in Beam Instrumentation Workshop 2000: Ninth Workshop, edited by K. D. Jacobs and R. C. Sibley, AIP Conf. Proc. No. 546 (AIP, New York, 2000), p. 267.

[5] R. E. Shafer, in Accelerator Instrumentation, edited by E. R. Beadle and V. J. Castillo, AIP Conf. Proc. No. 212 (AIP, New York, 1990), p. 26.

[6] S.S. Kurennoy, in Beam Instrumentation Workshop 2000: Ninth Workshop (Ref. [4]), p. 283; S. S. Kurennoy and R. E. Shafer, in Proceedings of the 7th European Particle Accelerator Conference (EPAC2000), Vienna, 2000 (Ref. [4]), p. 1768.

[7] S. S. Kurennoy, R. L. Gluckstern, and G. V. Stupakov, Phys. Rev. E 52, 4354 (1995).

[8] S.S. Kurennoy, in Proceedings of the 5th European Particle Accelerator Conference (EPAC96), Sitges, 1996 (Institute of Physics, Bristol, UK, 1996), p. 1449 (http:// accelconf.web.cern.ch/accelconf/e96/contents.html).

[9] This remark is due to a discussion with M. M. Blaskiewicz. 